\title{
Geometrization of the Schrödinger equation: Application of the Maupertuis principle to quantum mechanics
}

\author{
ANTONIA KARAMATSKOU* \\ Institut für Physik, Humboldt-Universität zu Berlin, \\ 12489 Berlin, Germany; Institut für Theoretische Physik, \\ Freie Universität Berlin, 14195 Berlin, Germany \\ a.karamatskou@gmail.com \\ HAGEN KLEINERT \\ Institut für Theoretische Physik, \\ Freie Universität Berlin, 14195 Berlin, \\ Germany; ICRANeT Piazzale della Repubblica, \\ 10 -65122, Pescara, Italy \\ $h . k @ f u$-berlin.de
}

\begin{abstract}
In its geometric form, the Maupertuis principle states that the movement of a classical particle in an external potential $V(x)$ can be understood as a free movement in a curved space with the metric $g_{\mu \nu}(x)=2 M[V(x)-E] \delta_{\mu \nu}$. We extend this principle to the quantum regime by showing that the wavefunction of the particle is governed by a Schrödinger equation of a free particle moving through curved space. The kinetic operator is the Weyl-invariant Laplace-Beltrami operator. On the basis of this observation we calculate the semiclassical expansion of the particle density.
\end{abstract}

PACS numbers: 98.80.Cq, 98.80. Hw, 04.20.Jb, 04.50+h

\section{INTRODUCTION}

The famous Maupertuis principle was discovered in 1741 by Pierre Louis Maupertuis, later refined by Hamilton and Jacobi, who also gave a geometric interpretation of the situation [1]. It laid the foundation to the geometric formulation of Newton's laws, and was an important stimulus for Einstein's general theory of relativity. In this note we want to point out that this geometric view of classical physics remains also valid in the quantum regime, i.e., the quantum mechanics of a particle in a potential $V(x)$ may be described alternatively by a Schrödinger equation in a general curved space with $D$ dimensions with the Maupertuis metric

$$
g_{\mu \nu}(x) \equiv 2 M[V(x)-E] \delta_{\mu \nu} .
$$

The Hamiltonian of this Schrödinger equation contains the Weyl-invariant (conformally invariant) version

$$
\Delta_{\mathrm{W}}=\Delta-\frac{1}{4} \frac{D-2}{D-1} R
$$

of the Laplace-Beltrami operator

$$
\Delta=g^{-1 / 2} \partial_{\mu} g^{1 / 2} g^{\mu \nu} \partial_{\nu} .
$$

Our result supplies us with an answer to an old, very fundamental problem left open by Einstein's classical equivalence principle. That principle states that the classical laws of motion of a point particle can be derived from a coordinate transformation in spacetime whose inertial forces simulate the gravitational forces at the position of the particle.

Since a quantum particle is always an extended object described by a wave packet, there could, in principle, be a correction term $\xi R$ proportional to the curvature scalar $R$, whose size $\xi$ is undetermined by the classical equivalence principle. The geometric method, which we shall call the Quantum Maupertuis Principle, fixes the size of the $R$-term.

The paper is structured as follows:

- The second section is devoted to the derivation of the Schrödinger equation of a particle in curved space, thereby fixing the above-mentioned extra $R$-term.

\footnotetext{
* present address: Center for Free-Electron Laser Science, Luruper Chaussee 149, 22761 Hamburg, Germany
} 
- In Sec. 3 we calculate the propagator in curved space.

- Sec. 4 contains the application of the Quantum Maupertuis Principle, where the particle density is calculated explicitly.

- The fifth section treats two explicit examples in the Maupertuis metric.

- A summarizing section and an appendix with calculations conclude the present note.

\section{FORMULATING QUANTUM MECHANICS IN CURVED SPACE}

Consider the classical eikonal of an arbitrary trajectory of a point particle moving in Euclidean space with a potential $V(x)$. It is given by the integral

$$
S(E) \equiv \int \sqrt{g_{\mu \nu}^{E}(x) d x^{\mu} d x^{\nu}}
$$

with the Maupertuis metric (1). It is a functional of the trajectory which may be parametrized with the help of an arbitrary variable $\lambda$ as $x^{\mu}(\lambda)$, and rewritten as

$$
S(E) \equiv \int d \lambda \sqrt{g_{\mu \nu}[x(\lambda)] \dot{x}^{\mu}(\lambda) \dot{x}^{\nu}(\lambda)} \equiv l
$$

The integral coincides exactly with the invariant length of the trajectory.

According to Maupertuis, the eikonal $S(E)$ is extremal for the classical trajectory, i.e., the classical orbit is a geodesic trajectory. If $\lambda$ is chosen to coincide with the invariant length $l$, the extremization produces the geodesic differential equation

$$
\frac{d^{2} x^{\delta}}{d l^{2}}+\Gamma_{\alpha \beta} \delta \frac{d x^{\alpha}}{d l} \frac{d x^{\beta}}{d l}=0
$$

where $\Gamma_{\mu \nu}^{\lambda}$ are the Christoffel symbols ${ }^{1}$

$$
\Gamma_{\mu \nu}^{\lambda}=\frac{1}{2} g^{\lambda \sigma}\left(\partial_{\mu} g_{\sigma \nu}+\partial_{\nu} g_{\mu \sigma}-\partial_{\sigma} g_{\mu \nu}\right)
$$

Inserting the metric (1) into this equation, we see that Eq. (6) is fulfilled if the trajectory follows the Newton equation $x^{\prime \prime \mu}=-\partial^{\mu} V$. (Here, the time is chosen as the non-affine parametrization parameter and the prime denotes the derivative with respect to it.)

The Maupertuis metric in Eq. (1) differs from the flat Euclidean metric $\bar{g}_{\mu \nu} \equiv \delta_{\mu \nu}$ only by a conformal factor

$$
\Omega^{2}(x) \equiv 2 M[V(x)-E] .
$$

It is therefore called conformally flat. Calculating the curvature quantities with this metric (cf. Appendix ) in $D$ dimensions one obtains the curvature scalar:

$$
R=\frac{1-D}{4}\left[\frac{2 \partial^{\mu} \partial_{\mu} V}{M(E-V)^{2}}+\frac{(D-6) \partial^{\mu} V \partial_{\mu} V}{2 M(E-V)^{3}}\right] .
$$

Consider now the quantum mechanics of the point particle of energy $E$ in the potential $V(x)$. It is described by the Schrödinger equation

$$
(\hat{H}-E) \psi(x) \equiv\left[\frac{\hat{\mathbf{p}}^{2}}{2 M}+V(x)-E\right] \psi(x)=0,
$$

\footnotetext{
${ }^{1}$ We use the index conventions of the textbooks 2,3 .
} 
where $\hat{\mathbf{p}} \equiv-i \hbar \nabla$. Using the metric (1), this can be rewritten as

$$
\left[\Omega^{-2}(x) \hat{\mathbf{p}}^{2}+1\right] \psi(x)=0
$$

or as

$$
\left(\hbar^{2} \Delta_{\mathrm{W}}-1\right) \psi(x)=0
$$

where $\Delta_{\mathrm{W}} \equiv \Omega^{-2}(x) \sum_{\mu} \partial_{x^{\mu}}^{2}$. It is easy to verify that this is equal to the Weyl-invariant combination in Eq. (2) of the Laplace-Beltrami operator [see Eq. (3)] and $R$. The equation linking $\xi$ to the conformal coupling is also known as the Penrose-Tagirov-Chernikov equation 4 .

Equation (12) is a simple but very fundamental result. The Maupertuis metric (1) governs not only the classical, but also the quantum mechanical dynamics, provided that the Laplace-Beltrami operator is extended to the Weyl-invariant form $(2)$.

\section{PROPAGATORS IN CURVED SPACE}

The advantage of the curved-space reformulation [i.e., the Schrödinger equation in the form of Eq. 12 rather than Eq. [10], is that, in curved space, the particle is without a potential. It is a free particle moving through the Maupertuis metric (1). For such movements, there exist well-developed methods of calculating quantum properties pioneered by Bryce DeWitt [5-7] who studied quantum field theoretical models in curved space. In particular, DeWitt has given a semiclassical expansion of the matrix elements of the resolvent operator

$$
\left\langle x|\hat{\mathcal{R}}| x^{\prime}\right\rangle \equiv\left\langle x\left|\frac{i \hbar}{\mathcal{E}-\hat{\mathcal{H}}}\right| x^{\prime}\right\rangle,
$$

where $\hat{\mathcal{H}}$ is a curved-space translation operator in some pseudotime parameter $\tau$, and $\mathcal{E}$ is the associated pseudoenergy. The pseudotime $\tau$ is commonly called Schwinger time or fifth time. Note that the physical time is eliminated in our considerations by the geometrization method employed. The principle of least action in the classical case fixes the trajectory of the particle and allows for different velocities along the path. In the quantum case the Schrödinger equation contains this information via the extra term which extends the Laplace-Beltrami operator to the Weylinvariant form.

The Green function $G\left(x, x^{\prime}\right)=\left\langle x|\hat{R}| x^{\prime}\right\rangle$ can be written as an integral

$$
G\left(x, x^{\prime}\right)=\int_{0}^{\infty} d \tau\left\langle x, \tau \mid x^{\prime}, 0\right\rangle
$$

over the pseudotime displacement amplitude

$$
\left\langle x, \tau \mid x^{\prime}, 0\right\rangle=\left\langle x\left|e^{-i(\hat{\mathcal{H}}-\mathcal{E}) \tau / \hbar}\right| x^{\prime}\right\rangle .
$$

For arbitrary $s=\tau-\tau^{\prime}$, the result is given by the expansion

$$
\left\langle x, \tau \mid x^{\prime}, \tau^{\prime}\right\rangle=\frac{D_{\mathrm{MV}}^{1 / 2}\left(x, x^{\prime}\right)}{(2 \pi i \hbar s)^{D / 2}} e^{i \sigma\left(x, x^{\prime}\right) / s \hbar} \sum_{n=0}^{\infty} a_{n}(i s / 2 \hbar)^{n},
$$

where

$$
D_{\mathrm{MV}}^{1 / 2}\left(x, x^{\prime}\right) \equiv g^{1 / 4}(x) \Delta_{\mathrm{MV}}^{1 / 2}\left(x, x^{\prime}\right) g^{1 / 4}\left(x^{\prime}\right)
$$

is the Morette-van Vleck determinant and $\Delta_{\mathrm{MV}}\left(x, x^{\prime}\right)$ has the expansion

$$
\begin{aligned}
& \Delta_{\mathrm{MV}}^{1 / 2}=1+\frac{1}{12} R_{\mu \nu} \sigma^{\mu} \sigma^{\nu}-\frac{1}{24} R_{\mu \nu ; \rho} \sigma^{\mu} \sigma^{\nu} \sigma^{\rho}+ \\
& \left(\frac{1}{288} R_{\mu \nu} R_{\rho \tau}+\frac{1}{360} R_{\mu \nu}^{\alpha \beta} R_{\alpha \rho \beta \tau}+\frac{1}{80} R_{\mu \nu ; \rho \tau}\right) \sigma^{\mu} \sigma^{\nu} \sigma^{\rho} \sigma^{\tau}+\ldots
\end{aligned}
$$


Note that this expression is stated in the form of an endpoint expansion, which means, that the derivatives are evaluated at the endpoint $x^{2}$. Here, $\sigma$ is the geodesic interval biscalar (cf. Appendix ). DeWitt allowed for the presence of an extra term $\xi R$ in addition to the Laplace-Beltrami operator $\Delta$ on the right-hand side of the Schrödinger equation [cf. Eq. 455]. Then he derived a recursion relation for the expansion coefficients [6]

$$
\begin{aligned}
\sigma_{\mu}\left(a_{0}\right)_{;}{ }^{\mu} & =0 \\
(n+1) a_{n+1}+\sigma_{\mu}\left(a_{n+1}\right)_{;}{ }^{\mu} & =\Delta_{\mathrm{MV}}^{-1 / 2}\left(\Delta_{\mathrm{MV}}^{1 / 2} a_{n}\right)_{; \mu}{ }^{\mu}-\xi R a_{n}
\end{aligned}
$$

whose lowest terms are

$$
\begin{aligned}
a_{1} & =\left(\frac{1}{6}-\xi\right) R \\
a_{2} & =\frac{1}{6}\left(\frac{1}{5}-\xi\right) R_{; \mu}{ }^{\mu}+\frac{1}{2}\left(\frac{1}{6}-\xi\right)^{2} R^{2} \\
& -\frac{1}{180} R_{\mu \nu} R^{\mu \nu}+\frac{1}{180} R_{\mu \nu \rho \sigma} R^{\mu \nu \rho \sigma} .
\end{aligned}
$$

With these coefficients at hand the expansion in Eq. (16) can be evaluated as a function of the curvature quantities.

It is interesting to note that the size of the factors found by DeWitt who allowed for a "quantum potential" in his short-time expansions is not universal. While in his article on "Dynamical theory in curved space" he had found a factor of 1/12 in order to identify the correct Hamiltonian in curved space [5], two-loop corrections to path integrals in curved space required a factor of $1 / 8$ [ . Furthermore, there have been other proposals of Schrödinger equations and the coefficients of the curvature scalar term in curved space (see Ref. 911 and references therein). This shows that the problem of finding the "correct" quantum mechanical equations of motion in curved space is quite a difficult task and brings many new questions with it.

\section{APPLICATION OF THE QUANTUM MAUPERTUIS PRINCIPLE}

We now come to the announced application of the quantum Maupertuis principle by calculating the particle density of the Schrödinger equation 10

$$
\begin{aligned}
\rho(x ; E) & \equiv\langle x|\delta(E-\hat{H})| x\rangle \\
& =\frac{1}{2 \pi \hbar}\left\langle x\left|\sum_{n} \operatorname{disc}\left(\frac{i \hbar}{E-E_{n}}\right)\right| x\right\rangle,
\end{aligned}
$$

where "disc" stands for the discontinuity across the cut in the energy plane. A simple algebra shows that

$$
\left\langle x|\hat{R}| x^{\prime}\right\rangle=\frac{1}{2}\left\langle x|\hat{\mathcal{R}}| x^{\prime}\right\rangle\left[V\left(x^{\prime}\right)-E\right]^{-1} .
$$

Now we insert the DeWitt expansion (16) which reduces for $x=x^{\prime}$ to

$$
\langle x|\hat{\mathcal{R}}| x\rangle=\frac{g^{1 / 2}(x)}{(2 \pi i \hbar)^{D / 2}} \sum_{n=0}^{\infty} a_{n}\left(-\partial_{m^{2}}\right)^{n} \int_{0}^{\infty} \frac{d s e^{-i m^{2} s / 2 \hbar}}{s^{D / 2}},
$$

where the integral is simply $\Gamma(1-D / 2)\left(m^{2}\right)^{D / 2-1}$, so that the sum on the right-hand side becomes

$$
\sum_{n=0}^{\infty} a_{n} \Gamma(n+1-D / 2)\left(m^{2}\right)^{D / 2-(n+1)} .
$$

\footnotetext{
2 See subsection 10.3.1 in the textbook 2
} 
To be used in Eq. (24) we must take Eq. (25) for $\xi=(D-2) / 4(D-1)$ and $m^{2}=1$ and evaluate $a_{n}$ with curvature terms of the Maupertuis metric (1):

$$
\begin{aligned}
& \langle x|\hat{\mathcal{R}}| x\rangle=\left(\frac{M}{2 \pi \hbar^{2}}\right)^{D / 2}\left\{\Gamma(1-D / 2)[V(x)-E]^{D / 2}\right. \\
& -\frac{\hbar^{2}}{12 M} \Gamma(3-D / 2) \partial_{\mu} \partial^{\mu} V(x)[V(x)-E]^{D / 2-2} \\
& \left.+\frac{\hbar^{2}}{24 M} \Gamma(4-D / 2) \partial_{\mu} V \partial^{\mu} V(x)[V(x)-E]^{D / 2-3}+\ldots\right\} .
\end{aligned}
$$

The result is valid for $V(x)>E$ where the metric is positive. For $E>V(x)$ we use the property $V-E=e^{\mp i \pi}(E-V)$ to find the discontinuity across the cuts. Remembering the extra factor $(V-E)^{-1}$ in Eq. 24 we obtain from the DeWitt expansion the particle density $\rho_{\text {DW }}(x ; E) \equiv\langle x|\delta(\mathcal{E}-\hat{\mathcal{H}})| x\rangle$ as

$$
\begin{aligned}
& \rho_{\mathrm{DW}}(x ; E)=\frac{1}{\pi}\left(\frac{M}{2 \pi \hbar^{2}}\right)^{D / 2} \sin \left(\frac{\pi D}{2}\right) \\
& \times\left[\Gamma(1-D / 2)(E-V)^{D / 2-1}\right. \\
& -\frac{\hbar^{2}}{12 M} \Gamma(3-D / 2)(E-V)^{D / 2-3} \partial_{\mu} \partial^{\mu} V \\
& \left.-\frac{\hbar^{2}}{24 M} \Gamma(4-D / 2)(E-V)^{D / 2-4} \partial_{\mu} V \partial^{\mu} V+\ldots\right] .
\end{aligned}
$$

Now we employ the reflection formula for Gamma functions $\Gamma(1-z) \Gamma(z)=\pi / \sin (\pi z)$

$$
\begin{aligned}
& \rho_{\mathrm{DW}}(E ; x)=\left(\frac{M}{2 \pi \hbar^{2}}\right)^{D / 2}\left[\frac{1}{\Gamma(D / 2)}(E-V)^{D / 2-1}\right. \\
& -\frac{\hbar^{2}}{12 M} \frac{1}{\Gamma(D / 2-2)}(E-V)^{D / 2-3} \partial_{\mu} \partial^{\mu} V \\
& \left.+\frac{\hbar^{2}}{24 M} \frac{1}{\Gamma(D / 2-3)}(E-V)^{D / 2-4} \partial_{\mu} V \partial^{\mu} V+\ldots\right] .
\end{aligned}
$$

This agrees with the expansion in $\hbar$ obtained from the original Schrödinger equation 10 for $E>V(x)[12$.

By virtue of the bilocal character of the DeWitt techniques in curved space, the expansion of $\langle x|\hat{\mathcal{R}}| x\rangle$ exists also for the off-diagonal matrix elements $\left\langle x|\hat{\mathcal{R}}| x^{\prime}\right\rangle$ which serves to find also the off-diagonal particle density $\rho\left(x, x^{\prime} ; E\right) \equiv$ $\left\langle x|\delta(E-\hat{H})| x^{\prime}\right\rangle$ beyond the result stated in the literature [13].

\section{TWO PHYSICAL SYSTEMS IN THE CURVED-SPACE FORMULATION}

The case $D=1$ and the harmonic oscillator

In one dimension, the expression (9) and the coefficient of the curvature term in Eq. (2) are invalid separately. Although the curvature in one dimension vanishes we see in the latter equation that the conformally invariant Laplacian is well defined and can be used to derive the semiclassical density of states as shown in Sec. . The expression for a particle of mass $M$ in a harmonic oscillator potential in one dimension reads

$$
\begin{aligned}
\langle x|\hat{\mathcal{R}}| x\rangle & =\frac{1}{\pi \hbar} \sqrt{\frac{M}{2}}\left\{\frac{1}{[E-V(x)]^{1 / 2}}-\frac{\hbar^{2} V^{\prime \prime}(x)}{16 M} \frac{1}{[E-V(x)]^{5 / 2}}\right. \\
& \left.-\frac{5 \hbar^{2}\left[V^{\prime}(x)\right]^{2}}{64 M}[E-V(x)]^{7 / 2}+\ldots\right\},
\end{aligned}
$$

where the prime indicates the derivative with respect to $x$. This can be compared immediately to the expression of the time evolution amplitude in the Eq. (4.255) in Ref. [2], where the semiclassical density of states

$$
\rho_{c l}=\frac{1}{\pi \hbar} \frac{M}{\sqrt{2 M(E-V)}}
$$


[cf. Eq. (4.254) in [2] has been factored out. Following the steps shown in the cited reference, we recover the time dependence of the series expansion of the following diagonal time evolution amplitude

$$
\left(x t_{a} \mid x t_{b}\right)=\frac{1}{\sqrt{2 \pi i \hbar / M}} \sqrt{\frac{\omega}{\sin (\omega t)}} \exp \left\{i \frac{M \omega}{\hbar}\left[\tan (\omega t / 2) x^{2}\right]\right\} .
$$

In this way we confirm that for the harmonic oscillator the correct solution for the time evolution amplitude is recovered by the Quantum Maupertuis Principle.

The next subsection raises an interesting question concerning the extra term that has to be added to the Schrödinger equation by treating the hydrogen atom.

\section{The hydrogen atom in the Maupertuis metric in momentum space}

As mentioned before, the extra $R$-term found above is not universal. This can be seen by comparing the result with the quantum mechanics of another system in curved space: the hydrogen atom in momentum space. It obeys a Schrödinger equation

$$
\left(\mathbf{p}^{2}+p_{E}^{2}\right) \Psi(p)=\frac{2}{r} \Psi(p)
$$

Here, $r$ is the radial coordinate and $p_{E}^{2}=-2 E$ (in natural units with $\hbar=a_{H}=E_{H}=1$, where $a_{H} \equiv \alpha^{2} \hbar / m_{e} c$ is the Bohr radius and $E_{H}=\alpha^{2} m_{e} c^{2}$ is the Rydberg energy). By analogy with the previous approach we rewrite (34) as

$$
\left\{\frac{1}{4}\left[\hat{r}\left(\mathbf{p}^{2}+p_{E}^{2}\right)\right]^{2}-1\right\} \Psi(p)=0
$$

and identify $\hat{r}^{2}$ with $-\sum_{\mu=1}^{D} \partial_{p_{\mu}^{2}}$. By re-ordering the operators we can express 35 as a differential equation

$$
\left(\frac{1}{2} \Delta_{p}-p_{E}^{2}+1\right) \Psi=0
$$

where $\Delta_{p}$ is now the Laplace-Beltrami operator in momentum space formed with the metric

$$
g_{i j}=\frac{2}{\left(\mathbf{p}^{2}+p_{E}^{2}\right)^{2}} \delta_{i j}
$$

which is again conformally flat. The associated curvature scalar is now $R=2 D(D-1) p_{E}^{2}$, so that Eq. (36) can be rewritten as

$$
\left(\frac{1}{2} \Delta_{p}-\frac{R}{2 D(D-1)}+1\right) \Psi=0
$$

Remarkably, the coefficient of the $R$-term in this momentum space problem does not correspond to the Weyl-invariant expression, where the subtracted $R$-term would have been $(D-2) R / 8(D-1)=R / 16$ for $D=3$.

\section{CONCLUSION}

We have applied the classical Maupertuis principle to quantum mechanics by formulating the Schrödinger equation of a particle in curved space via the Maupertuis metric. This procedure fixes the arbitrary extra term that has to be added to the Schrödinger equation. The quantum version of the Maupertuis principle allows for the derivation of the semiclassical particle density.

The result gives us the possibility of studying the quantum mechanics of an arbitrary potential problem using the well-developed techniques of curved-space quantum mechanics. Conversely, it permits us to understand questions about the quantum mechanics in curved space from the knowledge of Schrödinger theory in flat space.

However, our study shows that the extra term suggested by this principle is not universal, which raises further questions about the nature of a Schödinger type of equation in curved space. 


\section{Differential geometry in the Maupertuis metric}

In this short appendix we derive the necessary curvature quantities in the Maupertuis metric. The geometric properties of the space in the Maupertuis metric can be calculated directly as functions of $\Omega(x)=2 M[V(x)-E]$. We observe that under the Weyl transformation $\bar{g}_{\mu \nu}(x) \rightarrow g_{\mu \nu}=\Omega^{2}(x) \bar{g}_{\mu \nu}(x)$, the Christoffel symbols [cf. Eq. (7)] change like

$$
\Gamma_{\mu \nu}^{\lambda}=\bar{\Gamma}_{\mu \nu}^{\lambda}+\Omega^{-1}\left(\delta_{\nu}^{\lambda} \partial_{\mu} \Omega+\delta_{\mu}^{\lambda} \partial_{\nu} \Omega-\bar{g}^{\sigma \lambda} \bar{g}_{\mu \nu} \partial_{\sigma} \Omega\right)
$$

Because of this, the Riemann tensor defined by the covariant curl

$$
R_{\mu \nu \lambda}^{\sigma}=\partial_{\mu} \Gamma_{\nu \lambda}^{\sigma}-\partial_{\nu} \Gamma_{\mu \lambda}^{\sigma}-\Gamma_{\mu \lambda}^{\tau} \Gamma_{\nu \tau}^{\sigma}+\Gamma_{\nu \lambda}^{\tau} \Gamma_{\mu \tau}^{\sigma}
$$

is related to $\bar{R}_{\mu \nu \lambda}{ }^{\sigma}$ by

$$
\begin{aligned}
& R_{\mu \nu \lambda}{ }^{\sigma}=\bar{R}_{\mu \nu \lambda}{ }^{\sigma} \\
& +\left(2 \bar{g}_{\lambda[\nu} \delta_{\mu] \beta} \bar{g}^{\sigma \alpha}-2 \delta_{[\nu}^{\sigma} \delta_{\mu] \alpha} \delta_{\lambda \beta}+\delta_{[\nu}^{\sigma} \bar{g}_{\mu] \lambda} \bar{g}^{\alpha \beta}\right) \frac{\left(\partial_{\alpha} \Omega\right)\left(\partial_{\beta} \Omega\right)}{\Omega^{2}} \\
& +\left(\delta_{[\nu}^{\sigma} \delta_{\mu] \alpha} \delta_{\lambda \beta}+\bar{g}^{\sigma \alpha} \bar{g}_{\lambda[\mu} \delta_{\nu] \beta}\right) \frac{\bar{\nabla}_{\alpha} \bar{\nabla}_{\beta} \Omega}{\Omega},
\end{aligned}
$$

where $\bar{\nabla}_{\mu} v_{\nu} \equiv \partial_{\mu} v_{\nu}-\bar{\Gamma}_{\mu \nu}{ }_{\nu} v_{\lambda}$ stands for the covariant derivative, and $\bar{g}_{\lambda[\nu} \delta_{\mu] \beta} \bar{g}^{\sigma \alpha}$ is defined as the antisymmetrized expression $\bar{g}_{\lambda[\nu} \delta_{\mu] \beta} \bar{g}^{\sigma \alpha} \equiv \bar{g}_{\lambda \nu} \delta_{\mu \beta} \bar{g}^{\sigma \alpha}-\bar{g}_{\lambda \mu} \delta_{\nu \beta} \bar{g}^{\sigma \alpha}$. The Ricci scalar $R \equiv g^{\nu \lambda} R_{\mu \nu \lambda}^{\mu}$ is obtained from Eq. 40 as

$$
\begin{aligned}
R & =\frac{\bar{R}}{\Omega^{2}}-2(D-1) \bar{g}^{\alpha \beta} \frac{\bar{\nabla}_{\alpha} \bar{\nabla}_{\beta} \Omega}{\Omega^{3}} \\
& -(D-1)(D-4) \bar{g}^{\alpha \beta} \frac{\left(\partial_{\alpha} \Omega\right)\left(\partial_{\beta} \Omega\right)}{\Omega^{4}} .
\end{aligned}
$$

Inserting $\Omega(x)=2 M[V(x)-E]$ gives the curvature scalar in the form

$$
R=\frac{1-D}{4}\left[\frac{2 \partial^{\mu} \partial_{\mu} V}{M(E-V)^{2}}+\frac{(D-6) \partial^{\mu} V \partial_{\mu} V}{2 M(E-V)^{3}}\right],
$$

[cf. Eq. (9)]

\section{Derivation of the propagator in curved space}

In this appendix the time evolution amplitude is calculated in curved space. As mentioned in Sec. a pseudotime is introduced with respect to which displacements are evaluated. The pseudotime displacement amplitude

$$
\left\langle x, \tau \mid x^{\prime}, 0\right\rangle=\left\langle x\left|e^{-i \hat{\mathcal{H}} \tau / \hbar}\right| x^{\prime}\right\rangle .
$$

satisfies the Schrödinger equation

$$
i \hbar \partial_{\tau}\left\langle x, \tau \mid x^{\prime}, 0\right\rangle=\hat{\mathcal{H}}\left\langle x, \tau \mid x^{\prime}, 0\right\rangle
$$

with the boundary condition in $D$ dimensions

$$
\left\langle x, 0 \mid x^{\prime}, 0\right\rangle=\delta^{(D)}\left(x-x^{\prime}\right) .
$$

The Lagrangian treated by DeWitt is

$$
\mathcal{L}=\frac{1}{2} g_{\mu \nu}(x) \dot{x}^{\mu} \dot{x}^{\nu}
$$

This has the pseudotime Hamiltonian $\mathcal{H}=\frac{1}{2} g^{\mu \nu}(x) p_{\mu} p_{\nu} \equiv \frac{1}{2} p^{\mu} p_{\mu}$, where $g^{\mu \nu}(x)$ is the inverse of the metric $g_{\mu \nu}(x)$, and the action

$$
\mathcal{A}\left(x, x^{\prime} ; \tau-\tau^{\prime}\right)=\int_{x, \tau}^{x^{\prime}, \tau^{\prime}} d \tau \mathcal{L}=\frac{\sigma\left(x, x^{\prime}\right)}{\tau-\tau^{\prime}}
$$


where $\sigma\left(x, x^{\prime}\right) \approx \frac{1}{2} g_{\mu \nu}(x)\left(x-x^{\prime}\right)^{\mu}\left(x-x^{\prime}\right)^{\nu}+\ldots$ is the geodesic interval. The action depends on the pseudotime only via this ratio. This is a consequence of the "free motion" in the metric $g_{\mu \nu}(x)$.

From the Hamilton-Jacobi equations it follows that

$$
\begin{aligned}
\frac{\partial \mathcal{A}}{\partial x^{\mu}} & =p_{\mu}=\frac{\sigma_{\mu}}{\left(\tau-\tau^{\prime}\right)} \\
-\frac{\partial \mathcal{A}}{\partial \tau} & =\frac{\sigma\left(x, x^{\prime}\right)}{\left(\tau-\tau^{\prime}\right)^{2}}=\mathcal{H}=\frac{1}{2} p_{\mu} p^{\mu} .
\end{aligned}
$$

DeWitt gave the solution of the Schrödinger equation $(45)$ as a power series in $\tau$ for the Hamiltonian

$$
\hat{\mathcal{H}}=\frac{1}{2}\left(-\Delta+\xi R+m^{2}\right),
$$

with an arbitrary parameter $\xi$. For small $\tau$ and $x$ close to $x^{\prime}$, the solution is simply

$$
\left\langle x, \tau \mid x^{\prime}, \tau^{\prime}\right\rangle \approx \frac{D_{\mathrm{MV}}^{1 / 2}\left(x, x^{\prime}\right)}{(2 \pi i \hbar s)^{D / 2}} e^{i \sigma\left(x, x^{\prime}\right) / s \hbar},
$$

where $s \equiv \tau-\tau^{\prime}$ and $D_{\mathrm{MV}} \equiv \operatorname{det}\left[-\partial_{\mu} \partial_{\nu}^{\prime} \sigma\left(x, x^{\prime}\right)\right]$ is the Morette-van Vleck determinant [14, 15].

[1] A. Clebsch, Vorlesungen über Dynamik nebst fünf hinterlassenen Abhandlungen desselben, Karl Gustav Jakob Jacobi, G. Reimer, Berlin (1866).

[2] H. Kleinert, Path Integrals in Quantum Mechanics, Statistics, Polymer Physics, and Financial Markets. 5th ed., World Scientific (2009).

[3] H. Kleinert, Multivalued Fields in Condensed Matter, Electromagnetism, and Gravitation. World Scientific, (2008).

[4] R. Penrose in Relativity, Groups and Topology. Gordon and Breach, London, p. 565 (1964);

N.A. Chernikov, E.A. Tagirov, Quantum theory of scalar field in deSitter space-time, Ann. I. H. Poincare A 9, 109-141 (1968).

[5] B.S. DeWitt, Dynamical Theory in Curved Spaces. I. A Review of the Classical and Quantum Action Principles. Rev. Mod. Phys., 29(3), 377-397 (1957).

[6] B.S. DeWitt in Relativity, Groups and Topology II. North Holland Physics Publishing (1984).

[7] B.S. DeWitt, Dynamical theory of groups and fields. Les Houches (1965).

[8] B.S. DeWitt, The Global Approach to Quantum Field Theory, volume 1 of The International Series of Monographs on Physics. Oxford Science Publications, (2003).

[9] L. Parker, Path integrals for a particle in curved space, Phys. Rev. D, 19 (2), 438-441 (1979)

[10] K. Olaussen, Operator Ordering from Feynman Diagrams, Phys. Lett. A 113A (2), 63-65 (1985).

[11] K.S. Cheng, Quantization of a General Dynamical System by Feynmans Path Integration Formulation, J. Math. Phys., 13 (11), 1723-1726 (1972)

[12] See Eq. (4.262) in 2.

[13] See Eq. (4.266) in 2 .

[14] C. DeWitt-Morette, The semiclassical expansion, Ann. Phys. 97, 367-399 (1976).

[15] J.H. Van Vleck, The Correspondence Principle in the Statistical Interpretation of Quantum Mechanics, Proceedings of the National Academy of Sciences 2, 178-188 (1928). 